\title{
Miliary Tuberculosis with Tubercular Uveitis Presenting as Fever of Unknown Origin: A Case Report
}

\author{
ROKSANA PARVIN ${ }^{1}$, MAHBUB MUTANABBI ${ }^{2}$, SHAMIMA SHARMIN SHOVA ${ }^{3}$, MARIA KIBTIAR ${ }^{1}$, \\ FARZANA SHARMIN ${ }^{1}$
}

\begin{abstract}
:
Miliary tuberculosis is a form of disseminated tuberculosis, which is more frequent in immunocompromised patient. Ocular involvement of mycobacterium tuberculosis is also an uncommon presentation of disseminated tuberculosis. Tubercular uveitis is most frequent form of ocular tuberculosis. If treatment is delayed, it may cause loss of vision. In many case of the systemic TB with ocular involvement, there may be no eye symptoms in early stage. On the other hand, many cases of ocular TB may not have any evidence of systemic TB. Here we have described a case of miliary tuberculosis with ocular uveitis who initially presented with fever without any significant physical findings and later developed eye symptoms including diminished vision.
\end{abstract}

Keywords: Miliary Tuberculosis, Tubercular Uveitis

\section{Introduction}

Tuberculosis (TB) is the ninth leading cause of death worldwide and most common cause of infection related death worldwide above HIV infection ${ }^{1}$. Tuberculosis primarily involves the lungs and its distant lymphohematogenous spread results in the development of extra pulmonary TB (EPTB). Infrequently, intense systemic dissemination from the rupture of a Mycobacterium tuberculosis-laden focus into a vascular channel results in a morphologically characteristic form of disease known as miliary tuberculosis $^{2,3}$. Classic miliary TB is defined as millet like seeding in the lung evidenced on chest radiography. Miliary TB is potentially a lethal disease, if not rapidly diagnosed and treated ${ }^{2,3}$. However, commonly the term miliary TB is restricted to disseminated TB with miliary shadows on chest radiograph ${ }^{4}$. Miliary tuberculosis is less frequent and accounts for about $2 \%$ of all reported cases of tuberculosis and up to $20 \%$ of all extra-pulmonary tuberculosis cases ${ }^{5}$. Milliary tuberculosis is more common in immunocompromised patient. Recently prevalence of miliary tuberculosis has increased due to increasing frequency of HIV infection ${ }^{3,5}$.

1. Phase B, Resident, General Paediatrics, BSMMU, Dhaka.

2. Associate Professor, Department of Paediatrics, BSMMU, Dhaka

3. Registrar, Paediatrics, Apollo Hospital, Dhaka

Correspondence: Dr. Roksana Parvin, Phase B, Resident, General Paediatrics, Block-C, $1^{\text {st }}$ floor, BSMMU, Dhaka. Mob: +8801717410308 E-mail: roksana2parvin@gmail.com

Received: $30-10-2018$

Accepted: 24-06-2019
Ocular involvement of Mycobacterium tuberculosis is an uncommon presentation of miliary tuberculosis and is caused by hematogenous spread of organism to eye. In most of the reported cases of ocular TB, there was no evidence of other organ involvement ${ }^{6}$. Though ocular TB can involve any tissue of eye and orbit, inflammation of the uveal tract is the most common eye manifestation due to its high blood supply. Among tubercular uveitis, posterior uveitis (choroiditis) is more common ${ }^{7}$. Tubercular uveitis still represents a minority of uveitis cases in different part of world ranging from $0.5 \%$ in the USA to $11.4 \%$ in Iraq where TB is endemic ${ }^{6}$. A definitive diagnosis of ocular tuberculosis requires identification of acid fast bacilli in ocular fluid by PCR technique or acid fast staining or culture for mycobacteria or histologic examination of ocular biopsy specimen showing caseating granuloma ${ }^{6,8,9}$. However, it is difficult to obtain ocular specimen. In a patient with consistent ocular features, but without microbiologic conûrmation from the eye, the diagnosis of TB from another anatomic site can be helpful ${ }^{8}$. The early diagnosis of tubercular uveitis is very important as it is a condition threatening the vision.

The objective of this report is to describe an adolescent girl of miliary tuberculosis with ocular involvement (anterior uveitis) and to highlight the importance of keeping in mind the possibility of miliary TB with eye involvement in case of fever of unknown origin with eye complaint in an immunocompetent patient. 


\section{Case description:}

A 14-year old Bangladeshi adolescent girl, referred from another hospital admitted to BSMMU, Dhaka with the complaints of low grade fever with evening rise of temperature for 2 months. She had also history of anorexia and weight loss. There was no history of cough, respiratory distress, abdominal pain, joint pain, rash and contact with known pulmonary tubercular patient. She had also no significant past medical history or any associated comorbid condition. 5 years back, her mother was diagnosed to have tubercular lymphadenitis and was treated with anti-tubercular drugs \& improved.

Before attending to our hospital, the girl was treated with multiple oral \& parenteral antibiotics and also anti-malarial with adequate dose and duration and her condition remained static. On admission, she was very ill looking and physical examination revealed temperature $101^{\circ} \mathrm{F}$, vitals within normal limit \& there was no organomegaly. Her nutritional status was good and BCG mark was present. Respiratory system examination findings were respiratory rate$22 /$ min, symmetrical chest expansion, centrally placed trachea, resonant on percussion and normal vesicular breath sound with no added sound. We investigated her thoroughly keeping in mind the various cause of fever of unknown origin including tuberculosis. Complete blood count revealed normal leukocyte count and raised ESR ( $85 \mathrm{~mm}$ in $1^{\text {st }}$ hour). Peripheral blood film and Urine routine examination

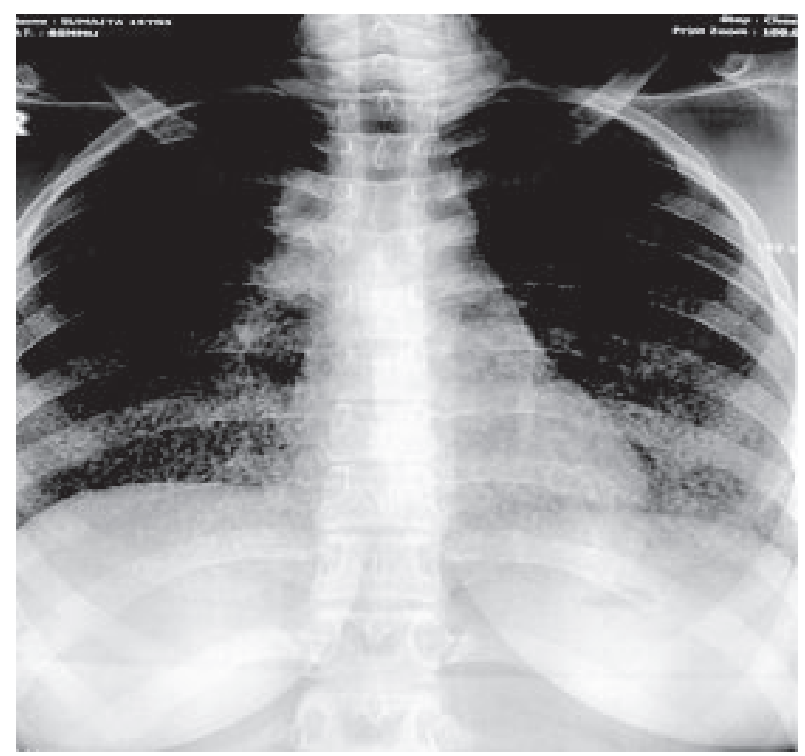

Fig.-1: Chest $X$ - Ray showing miliary shadow in both lung field result were within normal limit. Mantoux test induration size was $25 \mathrm{~mm}$ (positive). Induced sputum was negative for AFB and multiplex PCR did not detect any mycobacterium tuberculosis DNA in induced sputum. Chest X-ray showed widely distributed miliary shadow in both lung field. After the above mentioned evaluation, patient was diagnosed as a case of miliary tuberculosis.

At that time, before 2 days of starting anti-TB drugs, patient complaints about mild redness and watering from right eye. Anti-tubercular therapy with 4 drugs (rifampicin, isoniazid, pyrazinamide and ethambutol) was started. During anti-tubercular therapy, redness of right eye became more prominent on the next 2 days and at that time, it was associated with pain, photophobia, watering from right eye and impaired vision. Slit lamp examination of eye was done, which revealed oedematous cornea, mutton fat keratic precipitate and posterior synechia. There was also multiple tubercular nodule on iris. Intraocular pressure was also raised. Visual acuity was diminished to perception of hand movement. Ophthalmologist diagnosed it as tubercular uveitis (anterior). B scan of affected eye was also done and it revealed no abnormality in posterior segment but there was retrolental opacity with clear vitreous. Fundoscopy was not possible due to opacity in anterior chamber. Examination findings of opposite eye was normal. In view of vision threatening consequences, ophthalmologist suggested to give intra-ocular steroid immediately along with continuation of anti-tubercular drug. Patient and parents denied for that treatment. So, after consulting with ophthalmologist, patient was put on oral prednisolone (60 mg/ day) along with eye drop of atropine, topical steroid and acetazolamide.

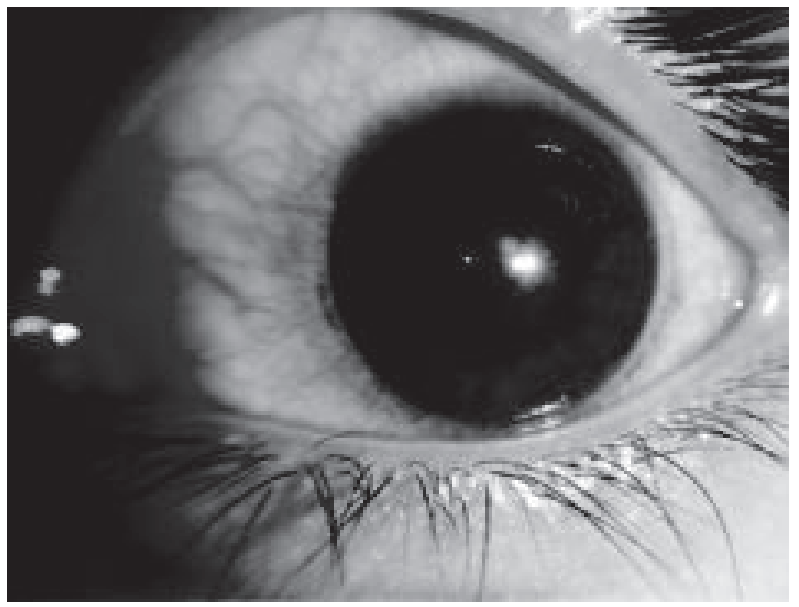

Fig.-2: Conjunctival congestion more marked around limbus 


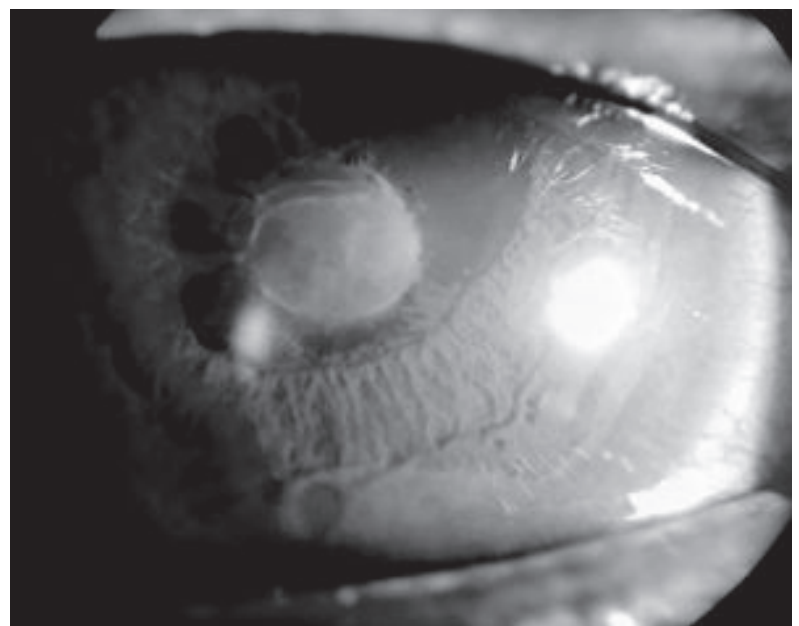

Fig.-3: Irregular pupillary margin and posterior synechiae on slit lamp examination

Patient was significantly improved with this treatment. On follow up after 7 days, eye pain, photophobia, watering and redness was absent. Vision also improved to $6 / 24$ on affected eye. After taking opinion from ophthalmologist, patient was discharged with anti-tubercular drugs and oral steroid for 4 weeks followed by tapering over 4 weeks. Patient was advised for regular follow-up and taking anti-TB drugs for 12 months ( 2 months intensive phase with 4 drugs and 10 months continuation phase with 2 drugs). On review after 1 month, patient was afebrile for 25 days, visual acuity improved to $6 / 12$ and general condition markedly improved. During that time, fundoscopy was done and it was normal. So, we advised for tapering of steroid.

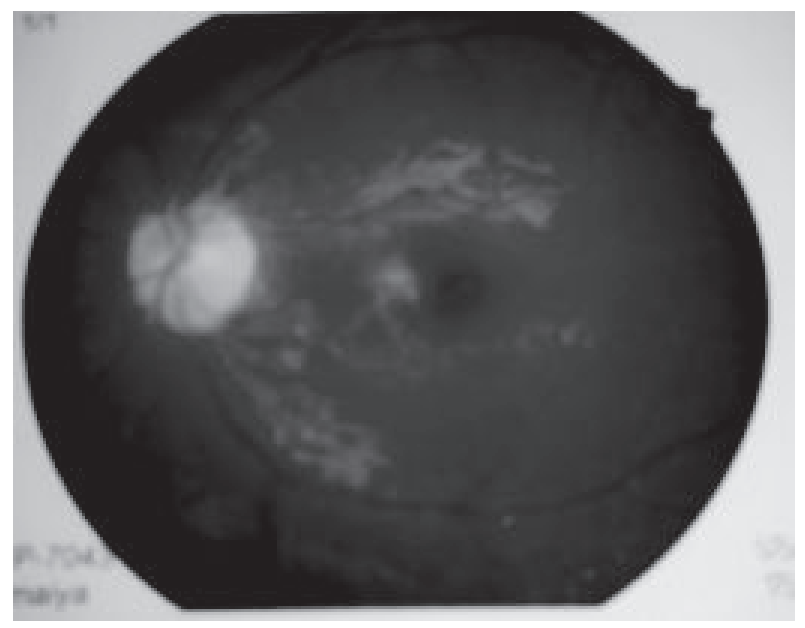

Fig.-4: Fundoscopic examination revealed normal retina on right eye

\section{Discussion:}

Worldwide burden of childhood tuberculosis is $6 \%$ of all tuberculosis cases ${ }^{10}$. In children, frequency of extrapulmonary TB is more than adult \& occur in 20$30 \%$ cases $^{10}$. But childhood tuberculosis is thought to be under reported because of paucibacillary disease. Miliary tuberculosis is potentially a lethal disease if not treated early. Although most cases of miliary tuberculosis are treatable, the mortality rate among children with miliary tuberculosis remains 15 to $20 \%{ }^{3}$. However, even in the endemic area, diagnosis of miliary tuberculosis is difficult due to atypical symptoms and nonspecific clinical signs. Patients with occult miliary TB can present with "pyrexia of unknown origin" without any localizing clue. So a high index of suspicion and a systematic approach is required for diagnosis 5,11 . In our patient, the complaints were low grade fever (occasionally high grade) for 2 months, anorexia and undocumented weight loss with good nutritional status and no response to multiple antibiotics. There was no lymphadenopathy and organomegaly. BCG mark was present. There was also no abnormal findings on respiratory system examination. So, she was diagnosed as fever of unknown origin before doing investigation. Mart et al. described that 50\% patient with miliary TB presented as fever of unknown origin $^{12}$. However, for diagnosis of disseminated TB in our patient, the negative points were absence of contact with pulmonary tubercular patient, no history of coughing or chest problem, presence of BCG scar, good nutritional status, absence of abnormal lung findings or lymphadenopathy and organomegaly. Mart et al. reported presence of hepatomegaly in $20 \%$ and splenomegaly in $19 \%$ patients among 269 adult diagnosed cases of milliary TB ${ }^{12}$. Although published series of childhood miliary TB are few, Sayantan et al. stated that lymphadenopathy and organomegaly in milliary tuberculosis are more common in children compared to adult ${ }^{5}$. Though the BCG protective efficacy is controversial ranging from 0 to $80 \%{ }^{13}$, miliary TB occur less often in children who received $B C G$ vaccine ${ }^{11}$. Hussey et al. found that $88 \%$ patient with miliary tuberculosis was BCG vaccinated ${ }^{14}$. Miliary TB is diagnosed by the presence of diffuse miliary infiltrate on chest radiograph or high-resolution computed tomography (HRCT) scan, or evidence of miliary tubercles in multiple organ. It is confirmed by bacteriological study, histopathology, and/or a dramatic chemotherapeutic response ${ }^{5}$. In complete 
blood count, anemia of chronic disease, leukocytosis, leucopenia, leukemoid reactions, and thrombocytopenia are some of the common abnormalities found in miliary TB. Erythrocyte sedimentation rate is usually elevated in patients with miliary TB $^{5}$. We found no change in blood count in several previous case report of miliary $\mathrm{TB}^{15,16}$. In our patient, complete blood counts were normal with high ESR. Induced sputum for AFB and multiplex PCR for M. tuberculosis was negative. Sensitivity of sputum smear for AFB is about $35 \%$ to $70 \%$, requiring 5000 to 10,000 bacteria/mL of sputum. It has less sensitivity in children due to paucibacillary disease ${ }^{17}$. Nucleic acid ampliûcation (NAA) methods may represent a signiûcant improvement for TB diagnosis. Tests based on NAA are rapid and usually highly speciûc for M. tuberculosis (close to 100\%). But in smear-negative sputum specimens, sensitivity of NAA is only $60 \%$ to $70 \%{ }^{18} .50 \%$ of miliary TB case may not have miliary mottling on chest $\mathrm{x}$-ray, where HRCT can be done if there is high degree of suspicion $^{6}$. In the present case, Chest $\mathrm{x}$-ray showed miliary mottling in both lung field, so HRCT was not done. Patients with miliary TB manifest tuberculin anergy more than those with pulmonary TB or EPTB, because disseminated disease usually occur in immunocompromised person. In various published pediatric study, tuberculin anergy has ranged from $35 \%$ to $74 \%^{5}$. However, Mantoux test was positive $(25 \mathrm{~mm})$ in our case.

In miliary tuberculosis, ocular involvement is not common in immunocompetent patient. The reported incidence of ocular TB is different in different parts of world. Lack of clear diagnostic criteria may explain the variation of the reported incidence of ocular TB over time and geographical location. The regions most affected by TB are also least equipped to screen thoroughly for ocular involvement, potentially resulting in underdetection of ocular manifestations [8]. The diagnosis of ocular TB is often presumed in the presence of suggestive ocular findings in combination with any of the following: systemic findings consistent with TB infection, positive interferon gamma release assay, positive tuberculin skin test in asymptomatic individuals. In addition, clinical response to anti-TB treatment (ATT) further supports a presumed diagnosis of ocular $\mathrm{TB}^{19}$. In a study, $10.7 \%$ patient of uveitis cases was found to be due to ocular TB in a specialized eye hospital in Bangladesh ${ }^{20}$. Most of them had no symptoms of other organ involvement.
They only presented with eye symptoms ${ }^{20}$. Anterior tubercular uveitis presents with redness, pain, raised IOP, mutton-fat keratic precipitates, photophobia, and ûoaters. Sometimes, iris nodules are found associated with anterior uveitis ${ }^{21}$. Our patient presented with mild redness of eye initially. Then gradually she developed pain, marked redness, photophobia, diminished vision. Ophthalmologic examination revealed oedematous cornea, mutton fat keratic precipitate, iris nodule, posterior synechia and visual acuity diminished to perception of hand movement. B scan of affected eye reveal clear vitreous. We could not do fundoscopy due to opacity in anterior chamber at initial diagnosis. But subsequently fundoscopy was done and it was normal. So, diagnosis was tubercular anterior uveitis. As there was no abnormality in posterior chamber, most probably visual acuity diminished due to opacity in ant. chamber and posterior synechiae. Treatment of tubercular uveitis are anti tubercular drugs along with intraocular or systemic steroid. In severe case, intraocular steroid is preferable. Recommended duration of antitubercular therapy for ocular TB vary in different studies. In a recent retrospective study, a duration of more than 9 months of anti-TB treatment was found to be associated with an 11-fold reduction in the likelihood of recurrence in patients with uveitis $^{22}$.

There is limited study on prevalence of systemic TB with ocular involvement. In a series of 100 randomly selected patients with culture-proven systemic TB without any eye symptoms seen in a university hospital in Spain, $18 \%$ were found to have intraocular $\mathrm{TB}^{23}$. So, in all patient with diagnosis of systemic TB, ophthalmologic evaluation should be done routinely.

\section{Conclusion:}

Miliary tuberculosis is a treatable condition. It is fatal if not diagnosed or treated early. However, diagnosis of miliary tuberculosis still remains a challenge because of atypical symptoms and clinical signs. So, a high index of suspicion and systematic approach are required for diagnosis. When miliary TB associated with ocular involvement, it is a vision threatening condition and require aggressive treatment. We should emphasize that whenever evidence of TB is found in any organ, other organ should be searched for evidence of tuberculosis especially in endemic area for tuberculosis. 


\section{References}

1. World health organization. Global tuberculosis report 2017, Geneva: World health organization; 1-147. https://www.who.int/tb/publications/ global_report/gtbr2017_main_text.pdf

2. Sahn SA, Neff TA. Miliary tuberculosis. American Journal of Medicine.1974; 56:495505

3. Sharma SK, Mohan A, Sharma A, Mitra DK. Miliary tuberculosis: new insights into an old disease. Lancet Infect Dis. 2005 Jul; 5(7):41530

4. Matsushima T. Miliary tuberculosis or disseminated tuberculosis. Internal Medicine. 2005; 44:687

5. Ray S, Talukdar A, Kundu S, Khanr D, Sonthalia $N$. Diagnosis and management of miliary tuberculosis: current state and future perspective. Therapeutics and Clinical Risk Management. 2015; 9: 9-26.

6. Shakarchi FA. Ocular tuberculosis: current perspectives. Clinical ophthalmology. 2015; 9:2223-2227

7. Helm CJ, Holland GN. Ocular tuberculosis. Surv Ophthalmol. 1993; 38:229-256

8. Bramante CT, Talbot EA, Rathinam SR, Stevens $R$, Zegans ME. Diagnosis of Ocular Tuberculosis: A Role for New Testing Modalities? International ophthalmology clinic. 2007 Summer; 47(3):45-62

9. Kurup SK, Chan CC. Mycobacterium-related ocular inûammatory disease: diagnosis and management. Ann Acad Med Singapore. 2006; 35:203-209

10. World health organization. Roadmap for childhood tuberculosis: Towards zero death, Geneva: WHO ; 2013

11. Sharma SK, Mohan A,Sharma A. Challenges in the diagnosis \& treatment of miliary tuberculosis. Indian J Med Res. 2012; 135:703730

12. Mert A, Arslan F, Kuyucu T, Koç EN, Yilmaz M, Turan D, Altin S. Miliary tuberculosis:
Epidemiologicaland clinical analysis of largecase series from moderate to low tuberculosis endemic Country. Medicine. 2017 FEB; 96(5):e5875

13. Thilothammal N, Krishnomurthy PV, Runyan DK, Banu K. Does BCG vaccine prevent tuberculous meningitis? Archives of disease of childhood. 1996 Feb; 74(2): 144-147

14. Hussey $\mathrm{T}$, Chisholm M, Kibel M. Miliary tuberculosis in children: a review of 94 cases. Pediatric infectious disease journal.1991. NOV; 10(11):832-6

15. Makoo ZB, Makoo RB, Mashrabi O. Miliary Tuberculosis: A Case Report. American Journal of Infectious Diseases. 2010; 6 (4): 103-106

16. Shaaban HA. Miliary TB with pelvic mass and choroidal tubercles in a child: Case report.Egypt Pediatr Assoc Gazette (2018), https://doi.org/ 10.1016/j.epag.2018.02.002

17. WHO. Laboratory Services in Tuberculosis Control Part II: Microscopy. Geneva, Switzerland: World Health Organization; 1998

18. Abe C, Hirano K, Wada M. Detection of Mycobacterium tuberculosis in clinical specimens by polymerase chain reaction and Gen-Probe Ampliûed Mycobacterium tuberculosis Direct Test. Journal of Clinical Microbiology. 1993; 31:3270-3274

19. Ocular tuberculosis- Asia pacific. American academy of ophthalmology. OCT, 2014.

20. Rahman Z, Ahsan Z, Rahman NA, Dutta MP . Pattern of Uveitis in a Referral Hospital in Bangladesh. Ocular Immunology and Inflammation. 2017(March); 26(6):1-4

21. Tabbara KF. Ocular tuberculosis: anterior segment. Int Ophthalmol Clin. 2005; 45:57-69

22. Ang M, Hedayatfar A, Wong W, Chee SP. Duration of anti-tubercular therapy in uveitis associated with latent tuberculosis: a casecontrol study. Br. J. Ophthalmol.2012; 96(3): 332-336

23. Bouza E, Merino P, Muñoz P, Sanchez-Carrillo $C$, Yáñez J, Cortés C. Ocular tuberculosis. A prospective study in a general hospital. Medicine (Baltimore). 1997; 76(1): 53-61. 